\title{
Vedic Multiplier Implementation for High Speed Factorial Computation
}

\author{
Prof. Sharayu Waghmare \\ Department of Computer Engineering, Bapurao Deshmukh College of Engineering Wardha India \\ sharayuwaghmare110@gmail.com
}

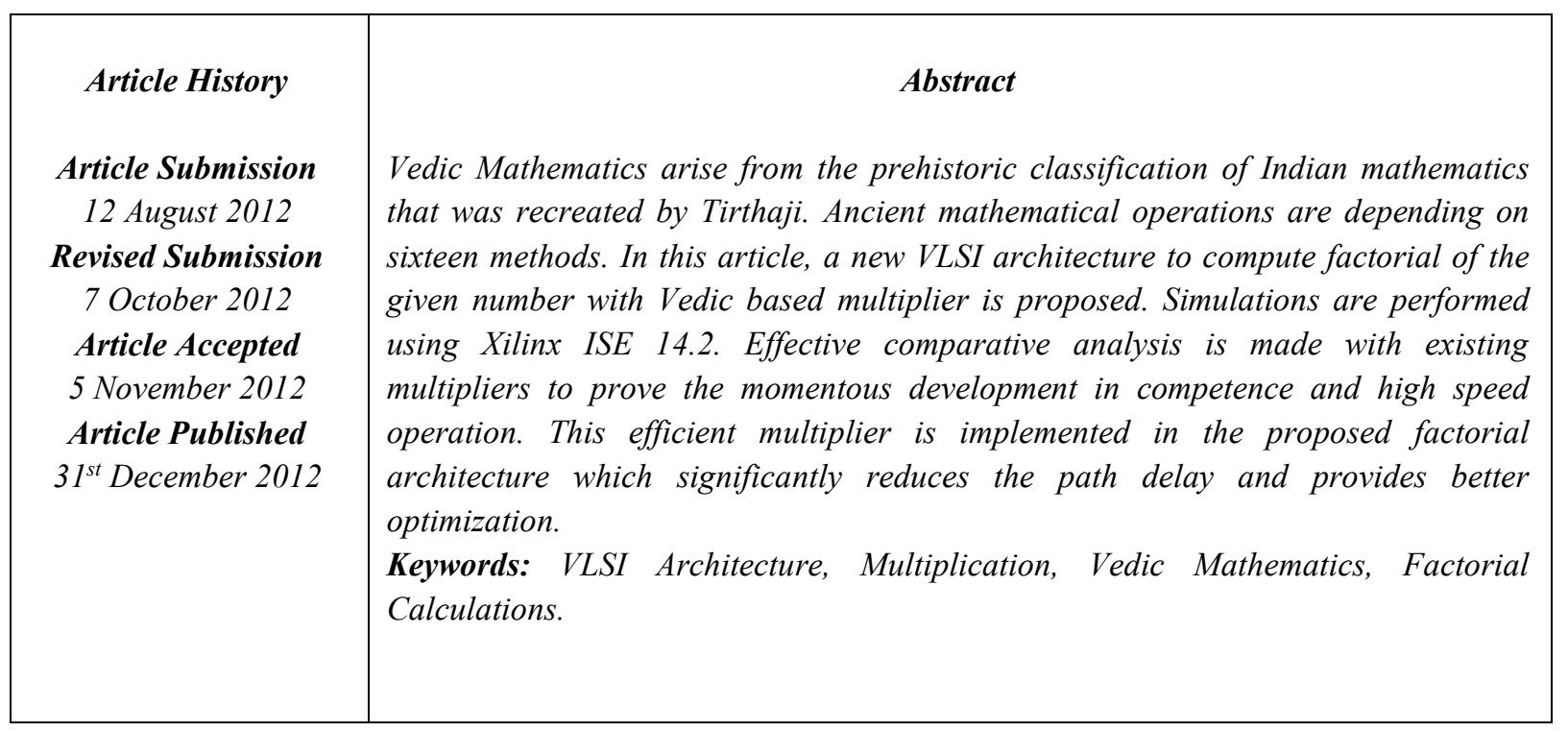

\section{Introduction}

Multipliers acts as heart of Microprocessors, Digital Signal Processors and high end communication oriented processors with sophisticated operational units. Adders plays a vital role in construction of high speed multipliers which are used to compute partial products. The necessity of high speed multipliers is directly proportional to the demand of real time processors for modern day operations. Dealing with reduced delay and power parameters are essential for domestic and industrial applications. Machine based multiplication is combination of addition and shifting operations [1].

Urdhava Tiryakbhyam Sutra is a method through which partial products are calculated in parallel which reduces the computation time there by enhancing its efficiency [4]. Conventional factorial results are compared with our proposed factorial architecture which has the efficient multiplier as one of its module which provides optimization in terms of speed and efficiency. When dealing with combinations and permutations (when dealing with probabilities), the importance of factorial is more [5].

\section{Vedic Multiplication}

U. Tiryakbhyam Sutra from vedic mathematics is used to perform multiplication in a faster way which means 'vertical as well as crosswise'. The steps concerned in the Urdhava Tiryakbhyam Sutra are as follows:

STEP1: It has n-bit Vedic multiplication unit.

STEP2: It has Partial products and carry.

STEP3: It has adders and the results of multiplication.

Consider a pair of two bit numbers such as a0a1 and b0b1. Multiplication process is as follows. The process of multiplication is shown in figure 1 and steps is shown in figure 2 . 


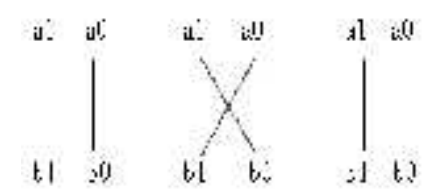

Fig.1 Multiplication of 252x846 (decimal)

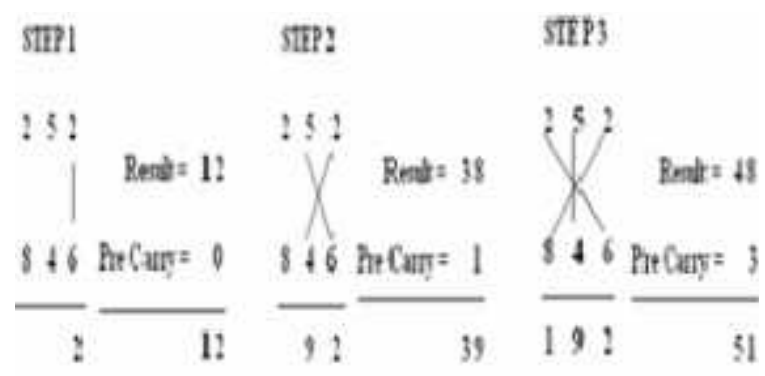

STIP!

STIPS

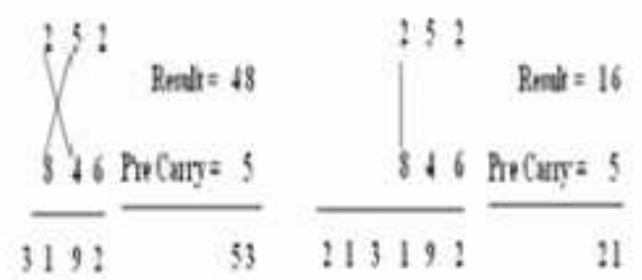

15: $: 846=21319$ ?

Fig.2 Steps in Multiplication of $252 x 846$ (decimals)

\section{Proposed Model of UT Multiplier to Calculate Factorial}

The architecture [2]-[3] shown in fig.1 is similar to conventional multiplier. Let's consider 2 bit numbers such as $\mathrm{A}=\mathrm{a} 1 \mathrm{a} 0$ and next $\mathrm{B}=\mathrm{b} 1 \mathrm{~b} 0$. The $2 \mathrm{X} 2 \mathrm{VM}$ module is premeditated using 4 AND gates and two half-adders. Architecture is as follows:

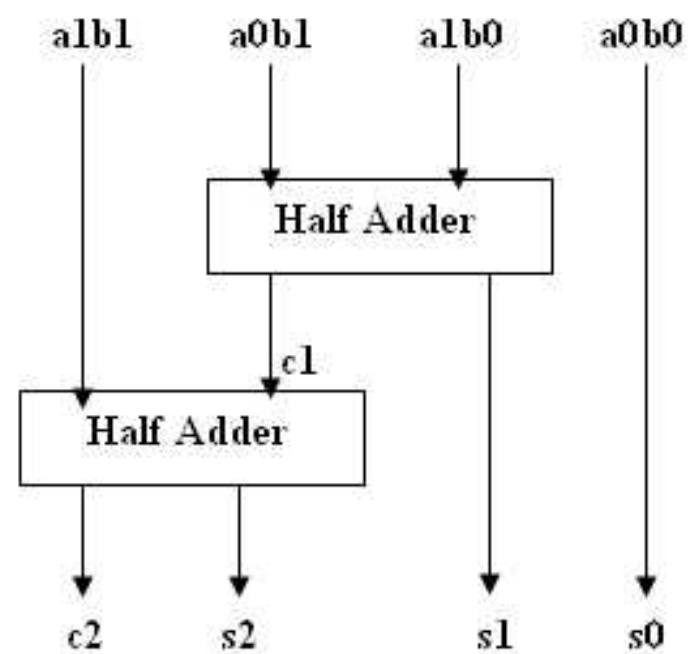

Fig.3. Architecture of $2 \times 2$ Vedic Multiplier 
With the four 2x2 bit VM modules, 4X4 VM is deployed in fig. 4. Let's consider a pair of 4-bit as A and B, say $\mathrm{A}=\mathrm{a} 3 \mathrm{a} 2 \mathrm{a} 1 \mathrm{a} 0$ \& $\mathrm{B}=\mathrm{b} 3 \mathrm{~b} 2 \mathrm{~b} 1 \mathrm{~b} 0$. The resultant product is 8 -bit- s7s6s5s4s3s2s1s0 .Architecture of $4 \mathrm{X} 4 \mathrm{VM}$ is shown in figure 4.

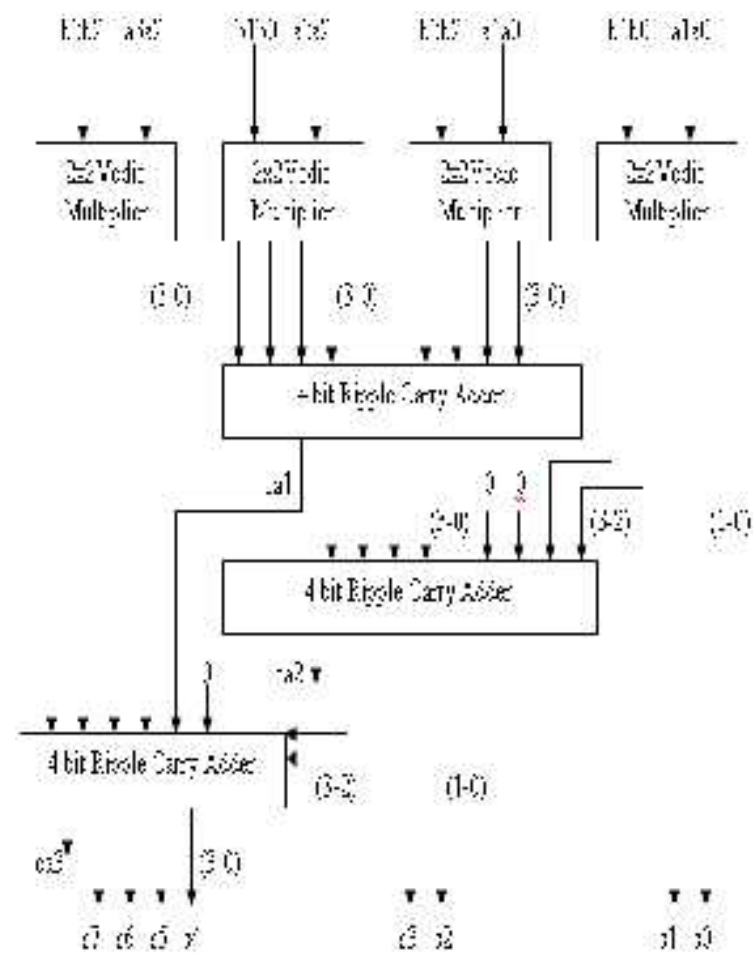

Fig.4. Block of $4 \times 4$ Vedic Multiplier

With the four $4 \mathrm{x} 4$ bit VM modules, $8 \mathrm{X} 8 \mathrm{VM}$ is deployed in fig. 5. Let's consider a pair of 8-bit as A and B. The resultant product is 16-bit-s15 to s0 .Architecture of $8 \mathrm{X} 8$ Vedic multiplier is exposed in figure 5.

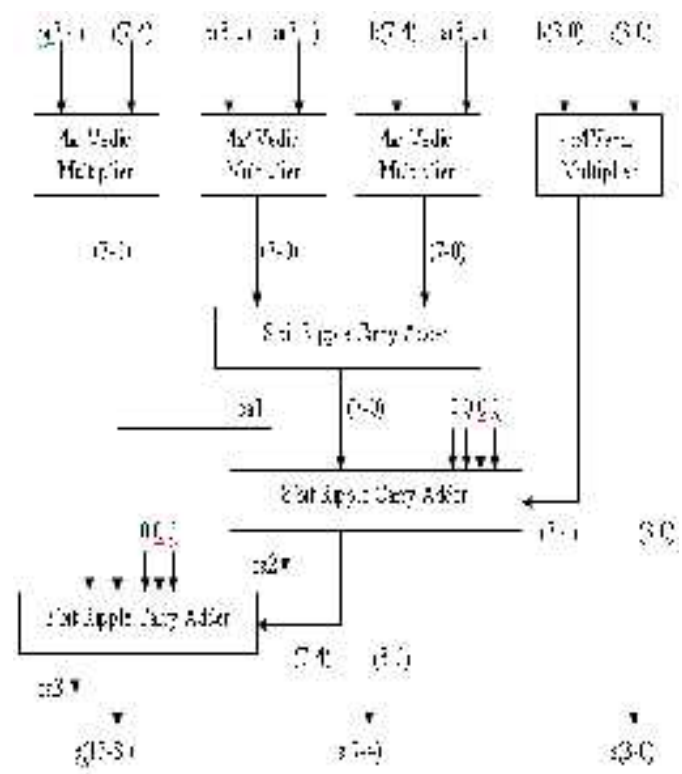

Fig.5. obstruct of $8 \times 8$ Vedic Multiplier 
With the four 8x8 bit VM modules, 16X16 VM is deployed in fig. 6. Let's consider a pair of 16-bit as A \& B. The resultant product is 32 -bit-s31 to s0 .Architecture of $16 \mathrm{X} 16 \mathrm{VM}$ is shown in figure 6 .

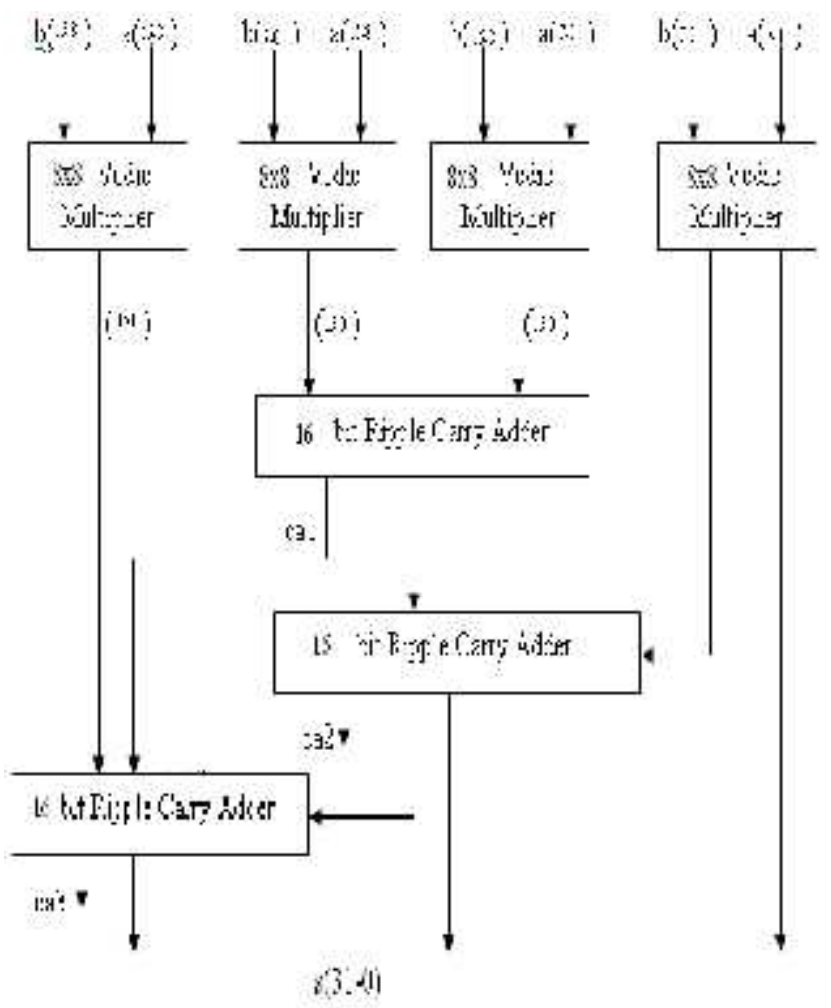

Fig.6. structural design of $16 x 16$ Vedic multiplier

\section{Proposed Architecture of Factorial Computation}

Factorial is the product of integers from 1 to $\mathrm{n}$ inclusive. The formula used to calculate the factorial is as follows:

Factorial of a number $=(n)(n-1)(n-2)$

Example: 4 ! Can be computed as follows:

$$
\begin{aligned}
4 ! & =(4) \times(4-1) \times(4-2) \times(4-3) \\
& =4 \times 3 \times 2 \times 1 \\
4 ! & =24
\end{aligned}
$$

We have used the efficient multiplier as one of the module to calculate the factorial of a number. The VM 16 bit multiplier is used to compute the factorial value. The architecture is explained as follows: First the number whose factorial to be computed is given as input ' $n$ '. The value of ' $n$ ' is stored in a memory. Up counter performs counting operation until its value reaches $n$. The output of up counter and temporary variable is repeatedly multiply up to $\mathrm{n}$ times. The architecture is shown in figure 7. 


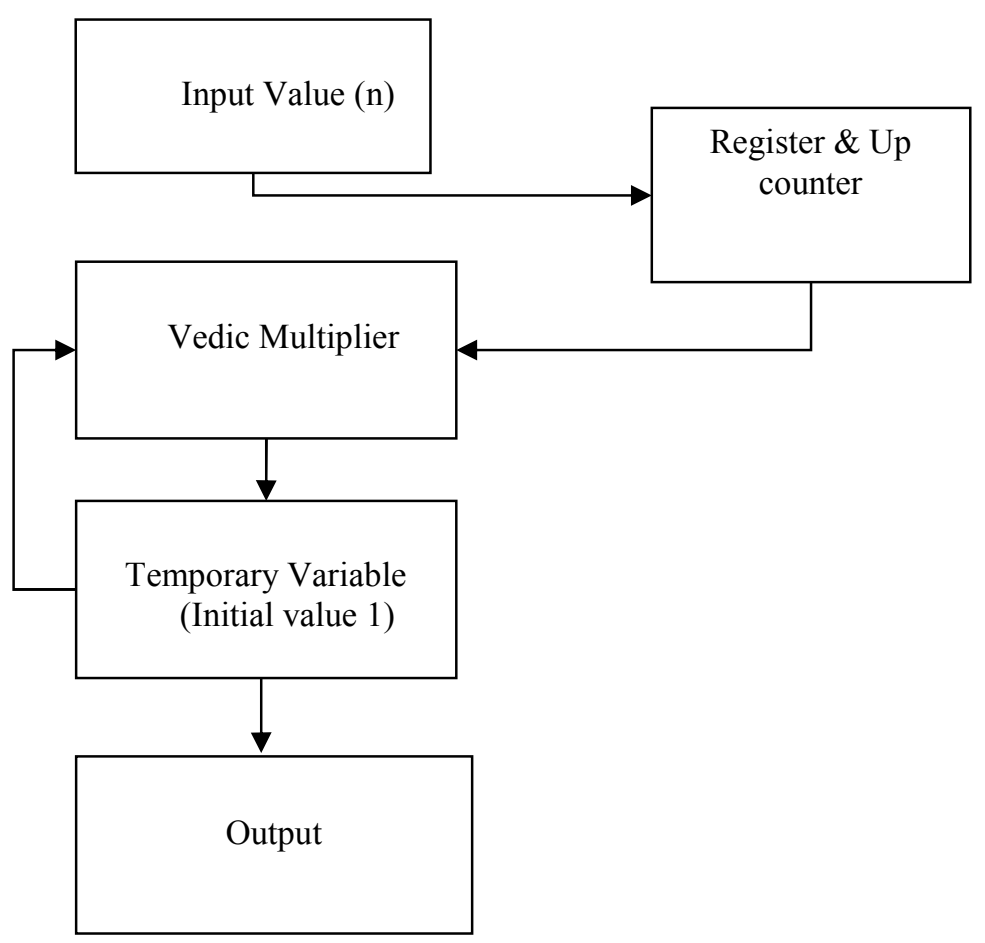

Fig.7. Block Diagram of Vedic Multiplier based Factorial Calculation

Simulations of the proposed design were conducted in the Xilinx ISE 14.2 design environment. The fig 8 \& 9 demonstrate the simulation results of 8 bit and 16 bit Vedic multiplier. Figure 10 shows the experimental results of factorial calculation using Vedic Multiplier.

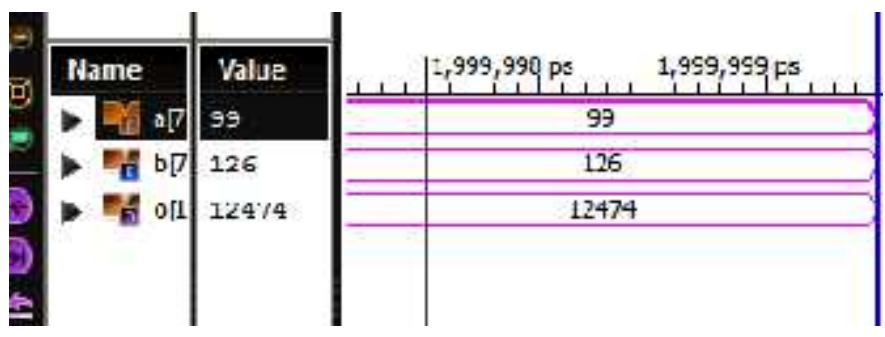

Fig. 8. Results-8x8 Vedic multiplier

\begin{tabular}{|c|c|c|c|}
\hline Name & Valiie & $L, 1,1$ & $1,99,99,1,1,1,399,99=08$ \\
\hline 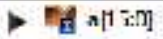 & 1028 & & 107.1 \\
\hline 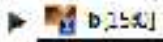 & 12000 & & 1.000 \\
\hline$\rightarrow[$ [ $031 x]$ & Iisteoo: & & $2 \pi 75000$ \\
\hline
\end{tabular}

Fig.9. results-16x16 Vedic multiplier

\begin{tabular}{|c|c|c|c|}
\hline Kane: & Whate & 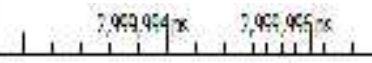 & $\mid 0,99:, 96: x$ \\
\hline Th & $100:$ & $1 \mathrm{M} 1$ & \\
\hline $10 \mathrm{he}$ & 101:::c100:: & $101100: 0015: 00 \%$ & \\
\hline
\end{tabular}

Fig. 10. Results-Factorial Calculation 
Table 1 depicts the comparative analysis of Vedic multiplier with conventional array multiplier for 8 and 16 bit, the synthesized results shows that VM outperforms in terms of delay compared to conventional multiplier.

Table I: Performance Analysis between Conventional and Vedic Multiplier

\begin{tabular}{|c|c|c|}
\hline Multipliers & $\begin{array}{c}\text { Number of } \\
\text { LUT's }\end{array}$ & $\begin{array}{c}\text { Delay } \\
\text { (ns) }\end{array}$ \\
\hline $\begin{array}{c}\text { Conventional Multiplier } \\
\text { 8x8 }\end{array}$ & 114 & 30.059 \\
\hline $\begin{array}{c}\text { Conventional Multiplier } \\
\text { 16x16 }\end{array}$ & 506 & 59.156 \\
\hline 8x8 Vedic Multiplier & 115 & 28.629 \\
\hline 16X16 Vedic Multiplier & 510 & 55.037 \\
\hline
\end{tabular}

\section{Conclusion}

The 2X2 VM module is designed using 4 number logic AND gates and 2 circuit based half adders. With the four number of $2 \times 2$ bit Vedic multiplier modules, $4 \mathrm{X} 4 \mathrm{VM}$ is deployed. With the four $8 \times 8$ bit Vedic multiplier modules, 16X16 VM is deployed. We have used the efficient multiplier as one of the module to calculate the factorial of a number. The VM 16 bit multiplier is used to compute the factorial value. The synthesized results shows that VM outperforms in delay compared to conventional multiplier. Using Vedic multiplication to calculate factorial is the better way to obtain optimized path delay and efficiency.

\section{References}

[1] Pushpalata Verma, K. K. Mehta , "Implementation of an Efficient Multiplier based on Vedic Mathematics Using EDA Tool "International Journal of Engineering and Advanced Technology (IJEAT) ISSN: 2249 - 8958, Volume-1, Issue-5, June 2012.

[2] Aniruddha Kanhe, Shishir Kumar Das and Ankit Kumar Singh, "Design and Implementation of Low Power Multiplier Using Vedic Multiplication Technique”, (IJCSC) International Journal of Computer Science and Communication Vol. 3, No. 1, pp. 131-132, January-June 2012.

[3] S.S.Kerur, Prakash Narchi, Jayashree C N, Harish M Kittur and Girish V A "Implementation of Vedic Multiplier For Digital Signal Processing" International conference on VLSI communication \& instrumentation (ICVCI), 2011.

[4] Sumita Vaidya and Deepak Dandekar, "Delay-Power Performance comparison of Multipliers in VLSI Circuit Design", International Journal of Computer Networks \& Communications (IJCNC), Vol.2, No.4, pp. 47-56, July 2010.

[5] Ramalatha, M.Dayalan, K D Dharani, P Priya, and S Deoborah, "High Speed Energy Efficient ALU Design using Vedic Multiplication Techniques", International Conference on Advances in Computational Tools for Engineering Applications (ACTEA) IEEE, pp. 600-603, July 15-17, 2009.

[6] P. Gulati, H. Yadav and M. K. Taleja, "Implementation of an efficient multiplier using the vedic multiplication algorithm," 2016 International Conference on Computing, Communication and Automation (ICCCA), Noida, 2016, pp. 1440-1443.

[7] Kanhe Aniruddha, Shishir Kumar Das, Ankit Kumar Singh, "Design and implementation of low power multiplier using vedic multiplication technique", International Journal of Computer Science and Communication, vol. 3, no. 1, pp. 131-132, 2012

[8] Shamim Akhter, VHDL Implementation of Fast NxN Multiplier Based on Vedic Mathematic, IEEE, 2007. 